\title{
MANUS Canada
}

\section{Abstracts presented at the Annual Meeting / Réunion annuelle} June 26, 2008

\section{2}

TREATMENT OF ACUTE NONDISPLACED OR MINIMALLY DISPLACED SCAPHOID WAIST FRACTURES: ARE CURRENT RECOMMENDATIONS FOLLOWED? EXPERIENCE OF A TERTIARY MEDICAL CENTER

Emilie Mailhot, Elia Botros, Marilou De Champlain, Jean-Paul Brutus

Montreal, Quebec

BACKGROUND: The standard treatment of nondisplaced or minimally displaced scaphoid waist fractures consisted originally in a long period of cast immobilization. In the last decade, treatment of these fractures has shifted towards early percutaneous screw fixation, a minimally invasive technique, thus avoiding cast immobilization and speeding the recovery. We therefore designed this study to review our current treatment protocol of acute nondisplaced or minimally displaced scaphoid waist fractures, in order to be able to offer recommendations for adequate treatment.

METHODS: Between January 2005 and December 2006, medical charts and X-rays of all patients presenting a scaphoid fracture were retrospectively reviewed. Patients' demographics, mechanism of injury, delay of treatment, type of imaging and treatment used as well as complications were documented.

RESULTS: A total of 128 patients were treated for scaphoid fracture. Forty-four patients were included $(31 \mathrm{M}, 13 \mathrm{~F})$ and had nondisplaced or minimally displaced scaphoid waist fractures. Average age of the patients was 36 years $(18-80$ years $)$.Thirty-six patients were treated with cast immobilization whereas eight patients had percutaneous screw fixation. The incidence of scaphoid non-union was $22.2 \%$ in the group treated by cast immobilization.

CONCLUSION: This study demonstrates that our treatment of acute nondisplaced or minimally displaced scaphoid waist fractures does not follow the current literature recommendations. Our incidence of non-union is significantly above that reported in the current literature. A great lack of appropriate imaging studies at the time of diagnosis and at follow up was noted. Our recommendations for a more appropriate treatment are proposed.

\section{3}

SCAPHOID NON-UNION: A RETROSPECTIVE ANALYSIS OF CASES TREATED WITH INTERNAL FIXATION AND CANCELLOUS BONE GRAFT COMING FROM GERDY'S TUBERCLE

Daniel Cloutier, Frédéric Arsenault

Quebec City, Quebec

INTRODUCTION: The management of scaphoid non-union remains controversial. Non-vascularized cancellous bone graft, traditionally harvested from the iliac crest or the distal radius, is a well-accepted treatment option for scaphoid's waist fracture. However, both techniques present limitations, respectively being pain at the donor site and the limited volume of bone available for grafting. The objective of our study is to demonstrate the advantages of harvesting from Gerdy's tubercle combined with internal fixation of the fracture.

METHOD: We reviewed ten cases of scaphoid non-union treated between 2006 and 2007. All patients had undergone non-vascularized cancellous bone graft harvested from Gerdy's tubercle and internally fixated with titanium screws. A team of occupational therapist assessed each patient before and after surgery on functional and symptomatic criteria and a radiological follow-up was also conducted.

RESULTS: The mean period of time between initial trauma and surgery was forty-two months (three and a half years). Three patients had avascular necrosis of the proximal pole, while three had previously undergone surgery. Clinical improvement, defined either as pain reduction, increased range of movement or radiological union, was achieved in seventy percent of cases. At two-week post-operatively, no patient showed discomfort or pain at the donor site.

CONCLUSION: We believe that non-vascularized cancellous bone grafting harvested from Gerdy's tubercle, combined with titanium screw fixation, is a valuable treatment option for scaphoid non-union. It provides a large volume of cancellous bone available for grafting and it is associated with minimal morbidity at the donor site. We plan to compare the longterm clinical outcome of our patients with others who had been treated using vascularized bone grafting.

OBJECTIVES: At the end of our presentation, plastic surgeons should understand the advantages of using Gerdy's tubercle as a potential donor site for the management of scaphoid non-union.

\section{4}

WHICH OUTCOME MEASURE IS THE BEST? EVALUATING RESPONSIVENESS OF THE MICHIGAN HAND QUESTIONNAIRE, THE DASH AND THE PATIENT SPECIFIC FUNCTIONAL SCALE FOLLOWING HAND AND WRIST SURGERY

\section{Paul Binhammer}

Toronto, Ontario

The objective was to evaluate and compare the responsiveness of 3 domain-specific outcome tools: the DASH, MHQ and PSFS in relation to 4 specific surgical situations of the hand. Subjects receiving surgical treatment for carpal tunnel syndrome, wrist pain, finger contracture or tumor, were given each questionnaire before surgery and 3 and 6 months after surgery. Responsiveness was denoted using standardized response means (SRM). Eighty-three patients completed the questionnaires at all 3 time points. When the sample was divided by diagnosis, the MHQ was the most sensitive of the three instruments for carpal tunnel and wrist pain patients from preop to 6 months and for tumor patients from preop to 3 months. The PSFS was the most responsive for those with finger contracture, exhibiting the highest sensitivity from preop to 6 months. All questionnaires exhibited low responsiveness between the 3- and 6- month followup visits. The DASH was not the most responsive instrument for any group over any time period. These results suggest that one questionnaire may be more appropriate for particular clinical problems.

\section{6}

\section{CONTRIBUTION OF THE ULNAR DIGITS TO THE GRIP STRENGTH OF THE HAND}

Saed Alhabib, Jennifer Methot, Robert Richards, Shrikant J Chinchalkar

Hand and Upper Limb Centre, London, Ontario

Grip strength is an objective measurement for assessment of hand function. The objective of this study is to determine the contribution of ulnar digits on grip strength in normal individuals.

One hundred normal hands were examined in 50 individuals with a mean age of 35 years. A calibrated JAMAR dynamometer was used to test subjects in 3 configurations: (1) entire hand, (2) exclusion of small finger, and (3) exclusion of small and ring fingers using generic hand based finger splints.

There was a 33\% decrease in over all grip strength with exclusion of the litthe finger and $55 \%$ decrease in strength with exclusion of the ring and little fingers. The data of this study suggest that the ulnar two digits play a significant role in over all grip strength and limitation of one or both ulnar digits adversely affects the hand function.

LEARNING OBJECTIVE: Assess factors affecting grip strength in the hand. 


\section{7}

THE WIDE AWAKE APPROACH TO DUPUYTREN'S DISEASE: FASCIECTOMY UNDER LOCAL ANAESTHESIA WITH EPINEPHRINE AND NO TOURNIQUET

Rebecca Nelson, Joseph Doumit, Amanda Higgins, Joanie Conrad, Phil Barnsley, Mike Bell, Don Lalonde

Halifax, Nova Scotia

PURPOSE: To show that Dupuytren's fasciectomy under local anaesthesia with epinephrine in the absence of tourniquet produces equivalent outcomes to fasciectomy under general anaesthetic with a tourniquet, with fewer risks to the patient.

METHOD: A multicenter retrospective review was conducted on 111 patients with Dupuytren's fasciectomies under local anaesthesia or general anaesthetic with tourniquet between 2001-2007. Data on patient demographics, comorbidities, cost, as well as pre- and post-operative range of motion was collected and evaluated using Microsoft Excel and SAS.

RESULTS/STATISTICS: A total of 148 fingers were treated, 102 under local and 46 under general anaesthetic. The group included 18 females and 93 males. The average postoperative TAM for individuals undergoing general anaesthetic was $199.0 \pm 29.6$ (D5), $223.9 \pm 29.3$ (D4), $234.6 \pm 14.6$ (D3) and $246.7 \pm 14.4$ (D2). The average postoperative TAM for individuals with local anaesthetic and epinephrine was $168.3 \pm 62.2 \mathrm{O}$ (D5), $195.9 \pm 67.5 \mathrm{O}$ (D4), $173.0 \pm 72.6 \mathrm{O}$ (D3), and $177.5 \pm 31.8 \mathrm{O}$ (D2). There were no significant differences between any of these groups $(\mathrm{p}=0.09$, $0.26,0.12$, and 0.20 respectively). Complication rates and types were similar with both techniques. The cost of performing fasciectomy in the clinic/office under local anaesthesia with epinephrine and no tourniquet was $\$ 36.46$ compared with $\$ 468.82$ in the main OR with tourniquet and no epinephrine. The yielded an estimated cost savings of $\$ 432.36$ when using local anaesthesia with epinephrine.

CONCLUSIONS: (1) The Wide Awake Approach to Dupuytren's contracture under local anaesthetic with epinephrine produces equivalent results to fasciectomies performed under a general anaesthetic. (2) Fasciectomy under local anaesthetic deletes general anesthetic risks and morbidities such as nausea and vomiting, and has cost benefits to health care providers.

LEARNING OBJECTIVE: To describe the benefits and drawbacks of performing Dupuytren's fasciectomy under local vs. general anaesthesia, considering patient outcomes and cost.

8

\section{THE FIRST 1400 CONSECUTIVE CASES OF WIDE AWAKE HAND SURGERY IN A SINGLE SURGEON'S PRACTICE: LESSONS LEARNED}

Don Lalonde, Jan Lalonde

St. John, New Brunswick

HYPOTHESIS: What were the most important lessons learned in the first 1400 consecutive cases of wide awake hand surgery in a single surgeon's practice?

METHODS: Wide awake hand surgery is performed with no sedation, no general anesthesia, and no tourniquet. Only lidocaine with epinephrine is injected directly into the affected parts of the hand and fingers for anesthesia and hemostasis. The author has kept careful records of all of the patients in his hand surgery practice in which $95 \%$ of surgery is now performed with the wide awake approach.

RESULTS: The first 1400 consecutive wide awake cases included 628 carpal tunnels, 167 trigger fingers, 129 operative reduction of hand fractures, 51 Dupuytren's, 34 extensor tendon repairs, 22 flexor tendon repairs, 11 trapeziectomies for basal joint arthritis, 6 tendon transfers and 2 tendon grafts.

SUMMARY: The wide awake approach has its greatest advantages in flexor and extensor tendon surgery (repairs, transfers, and grafting), in finger fracture surgery, and in complex secondary procedures because the surgeon can watch cooperative comfortable patients actively move reconstructed tendons and bones during the surgery and he can make adjustments to the repair before closing the skin. Carpal tunnels and trigger fingers can be performed with great convenience and efficiency for both the patient and the surgeon. Elderly patients or those on multiple medications avoid the risks and inconveniences of general anesthesia and hospital admission. However, repeat surgery for Dupuytren's contracture remains a challenge because of persisting bleeding in spite of epinephrine injection.

\section{9}

\section{ANALYSIS OF LIMITING WYNDELL-MERRITT SPLINT FOR EXTENSOR TENDON INJURIES TO HAND IMMOBILIZATION}

Michael W Neumeister MD, Nada Berry MD

Presenting Author: Michael W Neumeister MD

Corresponding Authors \& Address: Michael W Neumeister MD, SIU Plastic Surgery, PO Box 19653, Springfield, IL 62794-9653, USA. Ph: 217-545-7018, e-mail: mneumeister@siumed.edu with a cc to: cmatthews@siumed.edu

INTRODUCTION: The Wyndell-Merritt (relative motion) splint has been used effectively for extensor tendon lacerations. We have been treating extensor tendon injuries with the yoke splint without wrist immobilization with good clinical results. This study compares range of motion (ROM) of the injured finger to previously published results, as well as ROM for the digits treated with a traditional Wyndell-Merritt splint METHODS: A retrospective chart review was performed for two groups of patients treated over the last six years for complete extensor lacerations. Group A was treated with a relative motion hand splint only, while group $\mathrm{B}$ was immobilized at the wrist. Both groups of patients were treated with the same method of tendon repair, and were initially placed in a static splint. On follow-up, a controlled relative motion splint was fabricated and hand therapy commenced. The ROM figures were collected and analyzed. Two patients in group A were excluded and one patient was excluded from group B

RESULTS: Fourteen patients were included in group $A$ and 7 patients in group B. The average duration of treatment for both groups was 45 days. The average time of motion splint application for group A was 8.8 days post-operatively and 9.8 days for group B. Group A had 5 index, 7 long, 1 ring, and 2 small finger injuries that included 1 zone $I V, 11$ zone $V$, and 2 zone VI injuries. Group B had 3 index, 3 long, and 1 ring finger that included 2 zone IV, 3 zone $\mathrm{V}$ and 2 zone $\mathrm{VI}$ tendon lacerations. The ROM at conclusion of hand therapy was 230.4 and 230.7 degrees of flexion for groups A and B respectively. The average extensor lag for group A was 7.5 and 10.7 degrees for group B. One patient in each group required tenolysis for the complaint of skin adhesion.

CONCLUSION: Limiting the Wyndell-Merritt splint to the hand and not immobilizing the wrist has produced good clinical results for patients with complete EDC injuries in zones IV through VI. ROM for the patients treated with yoke splint only is the same as those treated with wrist splinting, and is comparable to previous studies with a traditional WyndellMerritt splint. The extensor lag is better for patients with a mobile wrist. These results support the use of a hand-based relative motion splint for treatment of zone IV, V, and VI extensor tendon lacerations.

\section{0}

\section{MANAGEMENT OF TENDON AVULSION AT THE MUSCULOTENDINOUS JUNCTION}

Jessica Colin-Durand, Achilleas Thoma

Hamilton, Ontario

PURPOSE: Since the literature lacks definite recommendations regarding musculotendinous junction tendon avulsions through digit amputations or closed injuries, we attempt to delineate optimal management through a case series and literature review.

METHOD: Seven cases of avulsion amputation with tendon avulsion at the musculotendinous junction were identified from the files of the senior author. A literature review yielded 24 cases of such amputations, and 17 cases of closed tendon avulsions. Patient demographics, method of injury, involved tendons, type of repair and outcomes were investigated.

RESULTS: Cases were mostly males aged 2 to 56 with work or sports injuries through rotation or longitudinal traction involving FDS, FDP, FPL, EPL, EIP, EDC, and EDM. Options included tendon resection, reat- 
tachment to muscle \pm encapsulation, side-to-side repairs, tendon transfers, and tendon grafts. Reattached to muscle were mostly flexors, side-to-side repairs were only in extensor-closed avulsions, while transfers were used in all instances. The thumb was treated with direct repairs or transfers. Amputations at DIP level had tendon resections, otherwise, options were similar whether the injury was closed or with an amputation. Follow-ups (7 weeks to 5 years) showed almost all patients returning to prior occupations. With amputations, joint motion was similar between reattachment and transfer, albeit with greater strength after transfers. For closed avulsions, results were slightly better with reattachment.

CONCLUSIONS: Several operative techniques are available for repair of such injuries. Certain types of tendons or digits may call for specific repairs, while level of amputation is also significant. The procedures in our series are similar to the literature except for our use of tendon grafts, which should also be considered.

\section{LEARNING OBJECTIVES:}

With this presentation, the learner will be able to:

1) Describe management options for musculotendinous junction avulsions 2) Identify whether certain tendons or digits respond better to specific procedures

3) Identify whether amputation level is significant for repair

\section{MANUS Author Index to Abstracts}

\section{A}

Alhabib S ............................6

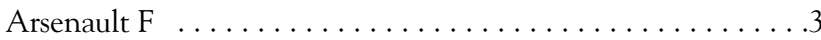

\section{B}

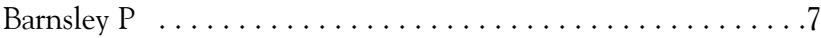

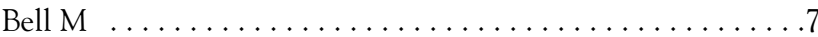

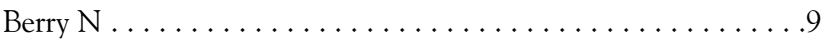

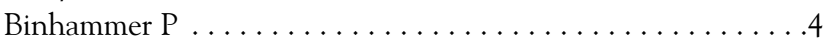

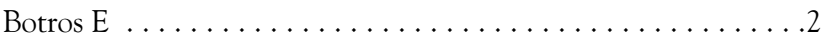

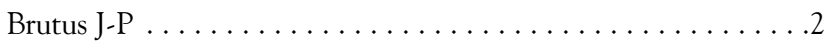

\section{$\mathrm{C}$}

Chinchalkar SJ .......................

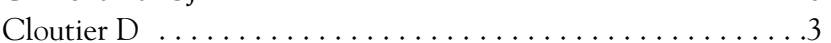

Colin-Durand $\mathrm{J} \ldots \ldots \ldots \ldots \ldots \ldots \ldots \ldots \ldots \ldots \ldots \ldots \ldots$

Conrad J . . . . . . . . . . . . . . . . . . . . . . .

$\mathrm{D}$

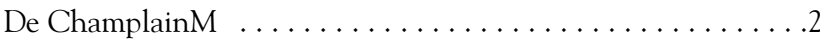

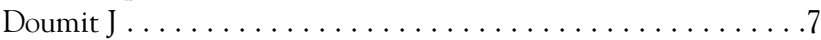

$\mathrm{H}$

Higgins $\mathrm{A} \ldots \ldots \ldots \ldots \ldots \ldots \ldots \ldots \ldots \ldots \ldots$

$\mathbf{L}$

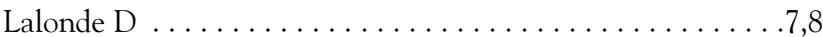

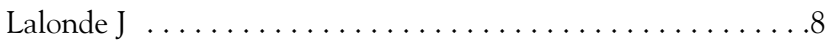

$\mathbf{M}$

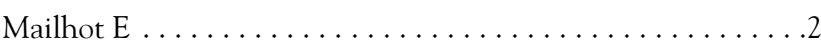

Methot J ..........................

$\mathrm{N}$

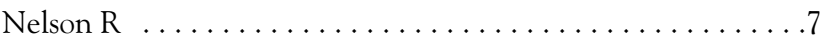

Neumeister MW $\ldots \ldots \ldots \ldots \ldots \ldots \ldots \ldots$

$\mathrm{R}$

Richards R

$\mathrm{T}$

Thoma A

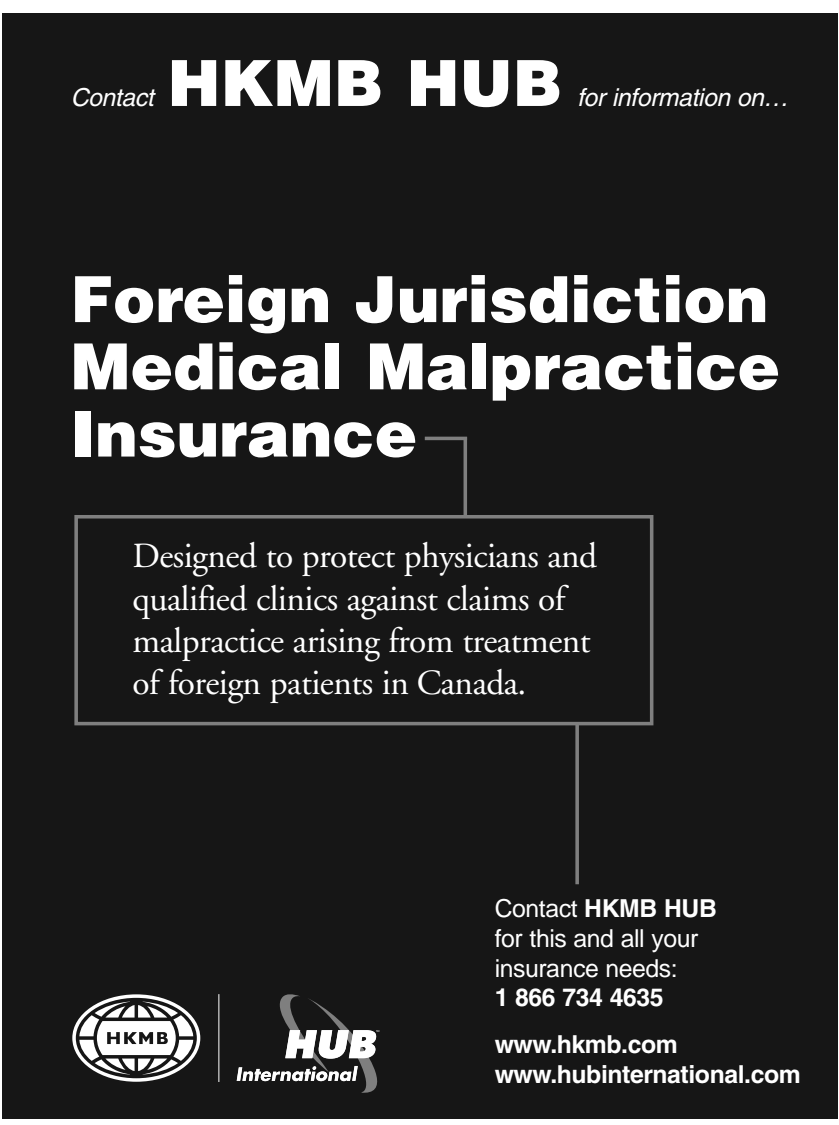

ADVERTISERS' INDEX

The Canadian Journal of Plastic Surgery is a peer-reviewed journal, financed almost entirely through advertising. The

companies advertising in the Journal recognize the need to support high-quality, Canadian, ethical journals and to promote the publishing of Canadian medical research.

The support of the following companies is appreciated:

Accurate Surgical \& Scientific Instruments

Perma Facial Implant Tunneler Passers and Forceps . . .64

Allergan Canada

Natrelle Collection . . . . . . . . . . . . . . . . OBC

Cardinal Health Canada

Snowden-Pencer Instruments . . . . . . . . . . . . . .62

Expand-a-Band Medical

Breast Binders . .....................132

HMKB International Insurance Brokers

Corporate ...........................

Leeches USA Ltd

Leeches . . . . . . . . . . . . . . . . . . . . . . . . . . . . . .59

Mentor Medical Systems Canada Inc

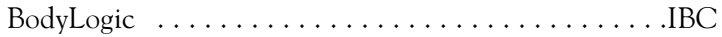

Oculo-Plastik, Inc

LaserSecure Shields . . . . . . . . . . . . . . . . 132

Stryker Canada

Craniofacial Symposium . . . . . . . . . . . . . . . . .60

Valeant Canada Limited

Dermatix $\ldots \ldots \ldots \ldots \ldots \ldots \ldots \ldots \ldots \ldots \ldots \ldots \ldots \ldots \ldots$

Young Pharmaceuticals

Corporate . . . . . . . . . . . . . . . .76 